\title{
Fibulin-3 serum and urine levels in the diagnosis and severity assessment of primary knee osteoarthritis
}

\author{
Mary Atef Nassif \\ Department of Physical Medicine, Rheumatology and Rehabilitation, Faculty of Medicine, Ain Shams University, \\ Cairo, Egypt
}

\begin{abstract}
Objectives: Osteoarthritis $(\mathrm{OA})$ is the most widespread joint disease and is a major cause of joint pain and disability in the middle aged and elderly population. The diagnosis of OA is based on clinical and radiographic changes that occur late after disease progression, and hence does not allow early detection of structural damage. Therefore, there is an acute need for reliable biochemical markers that can facilitate its earlier diagnosis. This study was commenced to identify fibulin-3 levels in serum and urine of patients with primary knee OA and to investigate their relationship with severity of the disease.

Material and methods: Fifty female patients with primary knee OA were compared to 25 healthy female controls. Fibulin-3 in serum and urine were measured using enzyme-linked immunosorbent assay (ELISA). Severity of knee pain was assessed by the Western Ontario and McMaster Universities Osteoarthritis (WOMAC) index and the Knee Outcome Survey Activities of Daily Living (KOS$\mathrm{ADL}$ ) scale. Radiographic grading of knee OA was performed by Kellgren-Lawrence (K-L) criteria.

Results: Serum and urine fibulin-3 were higher in patients $(286.00 \pm 145.00 \mathrm{ng} / \mathrm{ml}$ and 104.60 $\pm 32.73 \mathrm{ng} / \mathrm{ml}$, respectively) than in controls $(26.00 \pm 5.77 \mathrm{ng} / \mathrm{ml}$ and $13.60 \pm 4.21 \mathrm{ng} / \mathrm{ml}$, respectively). Fibulin-3 in serum and urine correlated $(p<0.001)$ with each other $(r=0.930)$ and with the severity of knee pain by WOMAC index ( $r=0.909$ and 0.928 , respectively), inversely correlated with KOS-ADL ( $r=-0.913$ and -0.953 , respectively), and with radiographic grading of K-L $(r=0.855$ and 0.875 , respectively).

Conclusions: Fibulin-3 serves as a biomarker of knee OA disease severity and could predict disease progression. Assessing urine fibulin-3 could be an applicable and easy method to diagnose knee OA and to follow up disease progression.
\end{abstract}

Key words: fibulin-3, radiography, primary knee osteoarthritis, WOMAC index.

\section{Introduction}

Osteoarthritis (OA) is the most widespread joint disease and is a major cause of joint pain and disability in the middle aged and elderly population. Its etiology is multifactorial (i.e. age, obesity, joint injury, or genetic susceptibility) [1]. All joints may be affected, but the knee is the most clinically significant site of primary $\mathrm{OA}$ involvement [2]. It is a progressive condition characterized by articular cartilage degeneration, subchondral bone sclerosis, osteophyte formation, and synovial inflammation [3].
Currently, the diagnosis of OA is based on clinical and radiographic changes that occur late after disease progression, and hence does not allow early detection of structural damage [4]. Therefore, there is an acute need for reliable biochemical markers that can facilitate the earlier diagnosis of OA and give an early warning of the initiation of matrix breakdown, prognosis, monitoring, and therapeutic strategies for the disease [4, 5]. Discovery of novel OA biomarkers is essential for early diagnosis, drug discovery, and to facilitate individualized treatment to improve clinical outcomes [6].

\section{Address for correspondence:}

Mary Atef Nassif, Department of Physical Medicine, Rheumatology, and Rehabilitation, Faculty of Medicine, Ain Shams University,

Cairo, Egypt, e-mail: yan_rheum@med.asu.edu.eg

Submitted: 9.08.2019; Accepted: 17.10.2019 
The fibulin family includes seven extracellular matrix (ECM) glycoproteins that are localized in basal membranes, stroma, and ECM fibres. Fibulins are thought to provide organization and stabilization to ECM structures during organogenesis and vasculogenesis. Their functions include mediation of cell-to-cell and cell-to-matrix communication, and elastic fibre formation, which is a specific function of the "elastic" fibulins 3, 4, and 5 [7].

Fibulin-3 is a member of a family of ECM proteins characterized by tandem array calcium binding (cb) of epidermal growth factor-like (EGF) domains and a C-terminal fibulin-type module [8]. Fibulin-3 epitopes (Fib 3-1, Fib 3-2, and Fib 3-3) contain a specific sequence of fibulin-3 [9, 10].

Electron microscopy of the recombinant fibulin-3 demonstrates a short rod structure with a globule at one end, which is probably the modified cbEGF domain. Under physiological conditions, fibulin-3 exists as monomers and it is highly conserved among different species with $92-94 \%$ of amino acids identical in human, rat, and mouse [7].

Human fibulin-3 is encoded by the gene EGF-containing fibulin-like extracellular matrix protein-1 (EFEMP1) located at chromosome 2p16. It contains 11 exons encoding a 493-amino acid protein with a molecular mass of 55 kilo-Dalton (kDa) [11].

During development, fibulin-3 is expressed in the mesenchyme, giving rise to cartilage and bone, indicating that fibulin-3 is important in skeletal development [11]. Also, fibulin-3 is expressed in cartilage and bone structures in the mouse embryo [11], and fibulin-3 peptides are potential biomarkers for the diagnosis of OA [9].

In adults, fibulin-3 is widely distributed in various tissue types, including the eye, and is highly expressed by epithelial and endothelial cells localized in their basement membranes. It is also present in blood vessels of different sizes and co-localized with fine elastic fibres. In addition, it is capable of inhibiting vessel development and angiogenesis both in vitro and in vivo [8].

Mice deficient in fibulin-3 (fibulin- $3^{-/-}$mice) show defects in elastic fibres and microfibrils [12-14]. These mice also exhibit reduced fertility and an early onset of aging-associated phenotypes, including decreased body mass, bone density, lordokyphosis, reduced hair growth, generalized fat, and muscle and organ atrophy [12].

Fibulin-3 interacts with another basement membrane protein, extracellular matrix protein 1 (ECM1). It also interacts with tissue inhibitor of metalloproteinase-3 (TIMP-3), collagen XVIII/endostatin, and elastin monomer tropoelastin. These interactions contribute to the integrity of basement membrane zones and anchor other ECM structures such as elastic fibres to basement membranes. Fibulin-3 stimulates the expression of TIMP-1 and TIMP-3 but inhibits the expression and activities of matrix metalloproteinase (MMP-2, MMP-3, and MMP-9). Dysfunction of basement membranes results in a wide variety of disorders from inflammation to cancer metastasis [7].

The aim of the present work was to investigate fibulin-3 levels in both serum and urine of patients with primary knee OA and study their relationship with severity of the disease.

\section{Material and methods}

Fifty female patients with the diagnosis of primary knee OA according to the American College of Rheumatology (ACR) criteria [15] were included, and 25 healthy females were also enrolled as controls in the study at the Department of Physical Medicine, Rheumatology and Rehabilitation, Ain Shams University Hospital.

Exclusion criteria included intraarticular injections within the last year, a previous lower extremity surgery, oral treatment with glucosamine and/or chondroitin within the last month, major-comorbidities, and inflammatory rheumatic diseases.

The study was carried out in accordance with the Code of Ethics of the World Medical Association (Declaration of Helsinki), the study was approved by the Ethics Committee of the hospital, and informed consent was obtained from subjects participating in the study.

\section{Clinical evaluation}

Patients were subjected to full medical history taking and thorough clinical examination.

\section{The Western Ontario and McMaster Universities Osteoarthritis index}

The Western Ontario and McMaster Universities Osteoarthritis (WOMAC) index is a three-dimensional, self-administered measure specific to OA, consisting of 24 items divided into three subscales: pain, stiffness, and physical function. The global score has a range from 0 (no symptom) to 96 (worst symptoms), with standardized score having a range from 0 to 100 [16].

\section{Knee Outcome Survey Activities of Daily Living scale}

The Knee Outcome Survey Activities of Daily Living (KOS-ADL) scale is a 14-item questionnaire in which each item is scored on a six-point Likert scale ( $0-5$ points), designed to evaluate symptoms and limitations during activities of daily living experienced within the last one to two days. The first six items assess symptoms such as pain, stiffness, swelling, instability, weakness, and limping. The next eight items assess subjects' function- 
al limitations during daily activities such as walking, ascending and descending the stairs, standing, squatting, kneeling, sitting with bent knees, and getting up from a chair. The maximum final score is 70 points, and it can be transformed to a $0-100 \%$ scale (where $100 \%$ indicates no symptoms and no limitation in daily activities) $[17,18]$

\section{Radiological assessment}

Plain radiographs anteroposterior weight bearing standing position for both knees were taken, and radiographic grading of OA was performed by using the Kellgren-Lawrence criteria (K-L) [19, 20].

\section{Measurement of fibulin-3 level assay}

Peripheral venous blood samples were collected in plain vacutainer tubes, and morning urine samples were collected in sterile urine containers from all participants in the study. All blood samples were allowed to clot for 10-20 minutes at room temperature and then were centrifuged for 20 minutes and sera were stored at $-20^{\circ} \mathrm{C}$ until analysis. Urine samples were transferred to sterile tube and were centrifuged for approximately 20 minutes, and until analysis the supernatants were stored at $-20^{\circ} \mathrm{C}$.

Serum and urine fibulin-3 levels were measured using an Enzyme-linked Immunosorbent Assay Kit (ELISA Kit for Human Fibulin 3 [FBLN3]; Bioassay Technology Laboratory, Shanghai, China). This kit uses an ELISA technique based on the Biotin double antibody sandwich technology to assay the Human Fibulin-3 (FBLN3).

\section{Statistical analysis}

Recorded data were analyzed using the Statistical Package for Social Sciences, version 20.0 (SPSS Inc., Chicago, Illinois, USA). Quantitative data were expressed as mean \pm standard deviation (SD). Qualitative data were expressed as frequency and percentage. Independent-samples t-test of significance was used when comparing two means. Pearson's correlation coefficient (r) test was used to assess the degree of association between two sets of variables. Receiver operating characteristic (ROC) curve analysis was used to find the best cut-off value with detection of sensitivity and specificity at this cut-off value. The confidence interval $(\mathrm{Cl})$ was set to $95 \%$ and the margin of error accepted was set to $5 \%$. So, the $p$-value was considered significant as follows: probability ( $p$-value) $<0.05$ was considered significant, $<0.001$ was considered as highly significant, and $>0.05$ was considered insignificant.

\section{Results}

Fifty female patients with primary knee OA complaining of knee pain were compared to 25 healthy females. Their age ranged from $45-70$ years with mean \pm SD of $58.30 \pm 8.05$ years for patients and 23-45 years (32.20 \pm 6.27 years) for the control group. Patients' BMI ranged from 28.4 to $49.44 \mathrm{~kg} / \mathrm{m}^{2}$ with mean $\pm S D$ of $39.28 \pm 6.22$ $\mathrm{kg} / \mathrm{m}^{2}$ and $22.65-47.08 \mathrm{~kg} / \mathrm{m}^{2}$ with $32.28 \pm 5.97 \mathrm{~kg} / \mathrm{m}^{2}$ for controls (Table I).

Mean duration of disease was $8.54 \pm 2.70$ years ranging from 3 to 12 years. 41 patients $(82 \%)$ had bilateral knee affection while nine (18\%) had unilateral affection. Mean

Table I. Comparison between patients and controls according to demographic data

\begin{tabular}{|c|c|c|c|c|}
\hline Demographic data & $\begin{array}{l}\text { Patients } \\
(n=50)\end{array}$ & $\begin{array}{l}\text { Control } \\
(n=25)\end{array}$ & $t$ & $p$-value \\
\hline Age (years) & & & 21.044 & $<0.001^{\star}$ \\
\hline Mean \pm SD & $58.30 \pm 8.05$ & $32.20 \pm 6.27$ & & \\
\hline Range & $45-70$ & $23-45$ & & \\
\hline Weight (kg) & & & 25.043 & $<0.001^{\star}$ \\
\hline Mean $\pm S D$ & $93.37 \pm 15.45$ & $75.96 \pm 11.22$ & & \\
\hline Range & 64-116.5 & 58-99 & & \\
\hline Height (cm) & & & 0.022 & 0.882 \\
\hline Mean \pm SD & $154.11 \pm 5.41$ & $153.92 \pm 4.75$ & & \\
\hline Range & $147-163$ & $145-162$ & & \\
\hline $\mathrm{BMI}\left(\mathrm{kg} / \mathrm{m}^{2}\right)$ & & & 21.68 & $<0.001^{*}$ \\
\hline Mean \pm SD & $39.28 \pm 6.22$ & $32.28 \pm 5.97$ & & \\
\hline Range & $28.4-49.44$ & $22.65-47.08$ & & \\
\hline
\end{tabular}

Independent sample t-test, $p$-value $>0.05$ not significant, * $p$-value $<0.001$ highly significant 
Table II. Comparison between patients and controls according to serum and urine fibulin-3

\begin{tabular}{|c|c|c|c|c|}
\hline Fibulin-3 & $\begin{array}{l}\text { Patients } \\
(n=50)\end{array}$ & $\begin{array}{l}\text { Control } \\
(n=25)\end{array}$ & $t$ & $p$-value \\
\hline Serum (ng/ml) & & & & $<0.001^{*}$ \\
\hline Mean \pm SD & $286.00 \pm 145.00$ & $26.00 \pm 5.77$ & & \\
\hline Range & $100-450$ & 20-100 & 91.774 & \\
\hline Urine (ng/ml) & & & & $<0.001^{*}$ \\
\hline Mean \pm SD & $104.60 \pm 32.73$ & $13.60 \pm 4.21$ & & \\
\hline Range & 50-150 & $10-50$ & 82.393 & \\
\hline
\end{tabular}

Independent sample t-test, * $p$-value $<0.001$ highly significant

WOMAC index was $55.38 \pm 10.37 \%$ ranging from 35.25 to $71.87 \%$ and mean KOS-ADL scale was $36.41 \pm 10.82 \%$ ranging from 16.25 to $52.5 \%$.

In patients, serum fibulin-3 was $286.00 \pm 145.00 \mathrm{ng} / \mathrm{ml}$, and it was $104.60 \pm 32.73 \mathrm{ng} / \mathrm{ml}$ in urine. In controls, fibulin-3 serum level was $26.00 \pm 5.77 \mathrm{ng} / \mathrm{ml}$, and in urine it was $13.60 \pm 4.21 \mathrm{ng} / \mathrm{ml}$. There was a significant difference between patients and controls as regards the serum and urine fibulin-3 ( $t=91.774,82.393$, respectively, and $p<0.001$ ) (Table II)

Radiographic data revealed different percentages for $\mathrm{K}-\mathrm{L}$ criteria in knee OA. About $36 \%$ of patients were grade G2, 44\% G3, and 20\% G4. Serum and urine fibulin-3 were significantly correlated with radiographic grading of K-L in knee OA ( $r=0.855$ and 0.875 , respectively, $p<0.001)$.

The serum fibulin-3 level was significantly correlated with the urine fibulin-3 level $(r=0.930, p<0.001)$. Also, the fibulin-3 level in both serum and urine was significantly correlated with disease duration $(r=0.795,0.718$, respectively, $p<0.001$ ).

Moreover, serum and urine fibulin-3 levels were significantly correlated with WOMAC index $(r=0.909,0.928$, respectively, $p<0.001$ ). The KOS-ADL scale was significantly inversely correlated with serum and urine fibulin-3 levels $(r=-0.913,-0.953$, respectively, $p<0.001)$. However, no significant correlation was found between age and serum ( $r=0.047, p=0.744)$ or urine fibulin-3 levels $(r=0.044, p=0.763)$.

Regarding the ROC curve: area under the curve (AUC) was 0.982 for serum fibulin-3 at a cut-off value > $35 \mathrm{ng} / \mathrm{ml}$, with sensitivity of $90 \%$ and specificity of $92 \%$. Also, AUC was 0.948 for urine fibulin-3 at a cut-off value $>20 \mathrm{ng} / \mathrm{ml}$, with sensitivity of $92 \%$ and specificity of $88 \%$.

\section{Discussion}

Fibulin-3 is important in skeletal development and widely distributed in various connective tissues including cartilage, bone, ligaments, and blood vessels [10].

This study showed a marked increase of fibulin-3 levels in both serum and urine of patients with knee
OA compared to control levels. Our findings suggest enhanced systemic production of fibulin-3, and the possible mechanism explaining that could reflect its release from degraded cartilage in primary knee OA.

It was revealed by Henrotin et al. [9] that the levels of two fragments of fibulin-3, called Fib 3-1 and Fib 3-2, were increased in urine and serum samples from $O A$ patients compared to healthy controls. Also, this was confirmed by cartilage immunostaining, which revealed that Fib 3-1 and Fib 3-2 were located in the extracellular matrix and cell clusters of the fibrillar zone but were absent in the surrounding un-fibrillated zones, indicating that fibulin-3 fragments measured in serum are generated, at least in part, in degraded cartilage. Also, Wu et al. [21] revealed that serum fibulin-3 concentrations were significantly higher in OA subjects than those in controls.

Indeed, fibulin-3 is not cartilage specific, and it is found in other tissue types, including bone. However, the immunohistochemical analysis by Henrotin et al. [9] showed that the concentrations of Fib 3-1 and Fib 3-2 were not increased in OA subchondral bone, suggesting that degradation of fibulin-3 in OA is a process that occurs mainly in cartilage.

In addition, de Visser et al. [6] showed that the increased Fib 3-3 serum concentration was positively correlated with the histological joint degeneration in the rat groove model of OA combined with metabolic dysregulation. This increase in Fib 3-3 was not only observed systemically in serum samples, but also locally with increased expression in the chondrocytes mostly in the superficial zone of the articular cartilage.

Because the fibulin-3 levels in serum and urine in our study were significantly correlated with the severity of clinical knee OA measures, assessed by WOMAC index and KOS-ADL scale, and radiological progression of the disease by K-L score, measurements of serum and/or urine levels of fibulin-3 might serve as a biochemical parameter for determining disease severity. Also, it might be predictive of prognosis with respect to the progression of the osteoarthritic disease process. 
This was in agreement with Runhaar et al. [4], who proved that all fibulin-3 epitopes were significantly associated to clinical knee OA measures. Conversely, none of them were significantly associated with the incidence of any of the radiographic knee OA measures.

However, Wu et al. [21] demonstrated that serum and synovial fluid fibulin-3 were correlated with the radiographic severity of $\mathrm{OA}$, which is evaluated by the $(\mathrm{K}-\mathrm{L})$ grading system, and stated that fibulin-3 could be also utilized as a biomarker for assessing the risk, severity, and progression of OA.

However, the role of fibulin-3 in the pathophysiology of OA and in the homeostasis of mature cartilage remains to be elucidated. Studies have confirmed that the overexpression of fibulin-3 suppresses chondrocyte differentiation by inhibition of cartilage node formation, proteoglycan production, and matrix gene expression [22].

Moreover, fibulin-3 is considered as a key elastogenic protein that controls the growth of elastic fibres in ligament. In particular, with other fibulins, fibulin-3 binds the monomeric form of elastin (tropoelastin) in vitro and has been shown to be involved in various aspects of elastic fibre development in vivo [23].

In early-stage OA, ligament laxity is commonly observed, leading to joint instability and then increased strain on joint tissue [24]. It is a major risk factor in the development of OA [25] because joint instability is a determinant of pain in OA [26]. Therefore, the degradation of fibulin-3 is associated with ligament laxity, joint instability, and subsequently with pain.

In our study, fibulin-3 serum levels were significantly correlated with urine fibulin-3 levels ( $r=0.930$, $p<0.001)$. To our knowledge, this is the first study to assess such a correlation between the serum and urine fibulin-3 levels. Estimation of urine fibulin-3 represents an easy and applicable method to diagnose and follow up disease progression.

In the present study, the area under the ROC curve was 0.982 for serum fibulin-3 and 0.948 for urine fibulin-3; it is used to estimate the ability of the biomarker to differentiate between patients and the healthy population to classify it as a diagnostic biomarker [27].

This was in agreement with Henrotin et al. [9], who stated that an acceptable diagnostic test is considered if the AUC value is $>0.75$. For serum levels of Fib 3-1 and Fib 3-2, AUC values were 0.751 and 0.838 , respectively, and serum Fib 3-2 can be considered as a potential diagnostic marker.

In our study, estimated sensitivity and specificity for serum fibulin-3 were $90 \%$ and $92 \%$, respectively, and for urine fibulin-3 they were $92 \%$ and $88 \%$, respectively. This was in accordance with Henrotin et al. [9], who es- timated that for Fib 3-1 assay, sensitivity was 68.4\% and specificity was $77.1 \%$, and for Fib 3-2 assay, sensitivity was $74.6 \%$ and specificity was $85.7 \%$.

In addition to the commercial availability of the ELISA assay, we measured the whole fibulin-3 molecule instead of its peptides because it is easier, more convenient, more cost-effective, and it ensures OA diagnosis if one peptide is more diagnostic than the others.

Further studies are needed to clarify if fibulin-3 plays a role in different inflammatory diseases affecting articular cartilage because the Kopeć-Mędrek and Kucharz study [28] revealed that there was no significant difference in its serum concentration measured in rheumatoid arthritis (RA) patients and healthy individuals.

Our patients and controls were females because knee OA is more frequent among women than men at any given age $>50$ years [1]. Also, fibulin-3 secretion can be regulated by estrogen [29, 30]. Henrotin et al. [9] revealed that hormonal status affect Fib 3-1 levels but not Fib 3-2 in healthy individuals.

To assess the effect of aging on fibulin-3 concentration, the mean age for patients in our study was 58.30 \pm 8.05 years, and for controls it was $32.20 \pm 6.27$ years. Fibulin-3 concentrations were higher in patients compared to controls, and no significant correlation was found between age and serum or urine fibulin-3 levels.

Our findings were in accordance to Henrotin et al. [9], who explained that the increase in Fib 3-1 and Fib 3-2 concentrations in OA patients compared to age-matched or younger healthy subjects is related to the disease itself, indicating that fibulin-3 epitope turnover is controlled in a different way in OA than in aging.

Limitations in our study included the small sample size with female predominance; a large sample size with comparison between female and male OA patients would better assess fibulin-3 levels with gender. Also, the controls in our study were younger, normal weight or overweight women, while the patients were older, overweight and obese females; matching controls would improve the age and BMI correlation with fibulin-3 levels.

In our research, assessment and correlation of fibulin-3 serum and urine levels with each other and with different aspects of primary knee OA including clinical evaluation, radiological assessment, and functional disability may enhance and refine its diagnostic and prognostic value.

\section{Conclusions}

Fibulin-3 serum and urine levels can serve as diagnostic biomarkers of knee OA, which may be helpful in assessing disease severity and prognosis. Measuring urine fibulin-3 levels can be an easy and applicable method for diagnosis and follow-up of knee OA. 


\section{Acknowledgements}

The author acknowledges Heba M. Adel, MD, Clinical Pathology; Faculty of Medicine, Ain Shams University for her help in the immunological assay of the study.

This research did not receive any specific grant from funding agencies in the public, commercial, or not-forprofit sectors.

The author declares no conflict of interest.

\section{References}

1. Martel-Pelletier J, Barr AJ, Cicuttini FM, et al. Osteoarthritis. Nat Rev Dis Primers 2016; 2: 16072.

2. Xu L, Zhu GB, Wang L, et al. Synovial fluid omentin-1 levels are inversely correlated with radiographic severity of knee osteoarthritis. J Investig Med 2012; 60: 583-586.

3. Pulsatelli L, Addimanda O, Brusi V, et al. New findings in osteoarthritis pathogenesis: therapeutic implications. Ther $\mathrm{Adv}$ Chronic Dis 2013; 4: 23-43.

4. Runhaar J, Sanchez C, Taralla S, et al. Fibulin-3 fragments are prognostic biomarkers of osteoarthritis incidence in overweight and obese women. Osteoarthritis Cartilage 2016; 24: 672-678.

5. Bay-Jensen AC, Henrotin Y, Karsdal M, et al. The need for predictive, prognostic, objective and complementary blood-based biomarkers in osteoarthritis (OA). EBioMedicine 2016; 7: 4-6.

6. de Visser HM, Mastbergen SC, Kozijn AE, et al. Metabolic dysregulation accelerates injury-induced joint degeneration, driven by local inflammation; an in vivo rat study. J Orthop Res 2018; 36: 881-890.

7. Zhang Y, Marmorstein LY. Focus on molecules: fibulin-3 (EFEMP1). Exp Eye Res 2010; 90: 374-375.

8. Albig AR, Neil JR, Schiemann WP. Fibulins 3 and 5 antagonize tumor angiogenesis in vivo. Cancer Res 2006; 66: 2621-2629.

9. Henrotin Y, Gharbi M, Mazzucchelli G, et al. Fibulin 3 peptides Fib3-1 and Fib3-2 are potential biomarkers of osteoarthritis. Arthritis Rheum 2012; 64: 2260-2267.

10. Henrotin Y, Sanchez C, Bay-Jensen AC, et al. Osteoarthritis biomarkers derived from cartilage extracellular matrix: current status and future perspectives. Ann Phys Rehabil Med 2016; 59: $145-148$

11. Ehlermann J, Weber S, Pfisterer P, et al. Cloning, expression and characterization of the murine Efemp1, a gene mutated in Doyne-Honeycomb retinal dystrophy. Gene Expr Patterns 2003; 3: 441-447.

12. McLaughlin PJ, Bakall B, Choi J, et al. Lack of fibulin-3 causes early aging and herniation, but not macular degeneration in mice. Hum Mol Genet 2007; 16: 3059-3070.

13. Rahn DD, Acevedo JF, Roshanravan S, et al. Failure of pelvic organ support in mice deficient in fibulin-3. Am J Pathol 2009; 174: 206-215.

14. Segade F. Molecular evolution of the fibulins: implications on the functionality of the elastic fibulins. Gene 2010; 464: 17-31.
15. Altman R, Asch E, Bloch D, et al. Development of criteria for the classification and reporting of osteoarthritis. Arthritis Rheum 1986; 29: 1039-1049.

16. Bellamy N, Buchanan WW, Goldsmith $\mathrm{CH}$, et al. Validation Study of WOMAC: A health status instrument for measuring clinically important patient relevant outcomes to antirheumatic drug therapy in patients with osteoarthritis of the hip or knee. J Rheumatol 1998; 15: 1833-1840.

17. Irrgang JJ, Snyder-Mackler L, Wainner RS, et al. Development of a patient-reported measure of function of the knee. J Bone Joint Surg Am 1998; 80: 1132-1145.

18. Bizzini M, Gorelick M. Development of a German version of the knee outcome survey for daily activities. Arch Orthop Trauma Surg 2007; 127: 781-789.

19. Kellgren JH, Lawrence JS. Radiological assessment of osteoarthrosis. Ann Rheum Dis 1957; 16: 494-502.

20. Felson DT, Naimark A, Anderson J, et al. The prevalence of knee osteoarthritis in the elderly. The Framingham osteoarthritis study. Arthritis Rheum 1987; 30: 914-918.

21. Wu Q, Suna X, Du L. Association of fibulin-3 concentrations with the presence and severity of knee osteoarthritis: A cross-sectional study. Knee 2017; 24: 1369-1373.

22. Wakabayashi T, Matsumine A, Nakazora S, et al. Fibulin-3 negatively regulates chondrocyte differentiation. Biochem Biophys Res Commun 2010; 391: 1116-1121.

23. Halper J, Kjaer M. Basic components of connective tissues and extracellular matrix: elastin, fibrillin, fibulins, fibrinogen, fibronectin, laminin, tenascins and thrombospondins. Adv Exp Med Biol 2014; 802: 31-47.

24. Sharma L, Lou C, Felson DT, et al. Laxity in healthy and osteoarthritic knees. Arthritis Rheum 1999; 42: 861-870.

25. Miura H, Takasugi S, Kawano T, et al. Varus-valgus laxity correlates with pain in osteoarthritis of the knee. Knee 2009; 16: 30-32.

26. Aunan E, Kibsgård TJ, Diep LM, et al. Intraoperative ligament laxity influences functional outcome 1 year after total knee arthroplasty. Knee Surg Sports Traumatol Arthrosc 2015; 23: 1684-1692.

27. Bauer DC, Hunter DJ, Abramson SB, et al. Classification of osteoarthritis biomarkers: a proposed approach. Osteoarthritis Cartilage 2006; 14: 723-727.

28. Kopeć-Mędrek M, Kucharz EJ. Fibulin-3 and other cartilage metabolism biomarkers in relationship to calprotectin (MRP8/14) and disease activity in rheumatoid arthritis patients treated with anti-TNF therapy. Adv Clin Exp Med 2018; 27: 383-389.

29. Blackburn J, Tarttelin EE, Gregory-Evans CY, et al. Transcriptional Regulation and Expression of the Dominant Drusen Gene FBLN3 (EFEMP1) in Mammalian Retina. Invest Ophthalmol Vis Sci 2003; 44: 4613-4621.

30. Hulleman JD, Brown SJ, Rosen $\mathrm{H}$, et al. A High-Throughput Cell-Based Gaussia Luciferase Reporter Assay for Identifying Modulators of Fibulin-3 Secretion. J Biomol Screen 2013; 18 : 647-658. 